\title{
Testing Gaussianity on Archeops data
}

\author{
A. Curto ${ }^{1,2}$, J. Aumont ${ }^{3}$, J. F. Macías-Pérez ${ }^{3}$, E. Martínez-González ${ }^{1}$, R. B. Barreiro ${ }^{1,2}$, D. Santos ${ }^{3}$, \\ F. X. Désert ${ }^{4}$, and M. Tristram ${ }^{3}$
}

\author{
1 Instituto de Física de Cantabria, CSIC-Universidad de Cantabria, Avda. de los Castros s/n, 39005 Santander, Spain \\ 2 Dpto. de Física Moderna, Universidad de Cantabria, Avda. los Castros s/n, 39005 Santander, Spain \\ 3 Laboratoire de Physique Subatomique et de Cosmologie, 53 avenue des Martyrs, 38026 Grenoble Cedex, France \\ ${ }^{4}$ Laboratoire d'Astrophysique, Obs. de Grenoble, BP 53, 38041 Grenoble Cedex 9, France \\ e-mail: reprints@archeops.org
}

Received 5 December 2006 / Accepted 16 July 2007

\section{ABSTRACT}

\begin{abstract}
Aims. We performed a Gaussianity analysis using a goodness-of-fit test and the Minkowski functionals on the sphere to study the measured Archeops Cosmic Microwave Background (CMB) temperature anisotropy data for a $143 \mathrm{GHz}$ Archeops bolometer. We consider large angular scales, greater than 1.8 degrees, and a large fraction of the North Galactic hemisphere, around $16 \%$, with a galactic latitude $b>15$ degrees.

Methods. The considered goodness-of-fit test, first proposed by Rayner \& Best (1989, Smooth Tests of Goodness of Fit), was applied to the data after a signal-to-noise decomposition. The three Minkowski functionals on the sphere were used to construct a $\chi^{2}$ statistic using different thresholds. The former method was calibrated using simulations of Archeops data containing the CMB signal and instrumental noise in order to check its asymptotic convergence. Two kind of maps produced with two different map-making techniques (coaddition and Mirage) are analysed.

Results. Archeops maps for both Mirage and coaddition map-making, are compatible with Gaussianity. From these results we can exclude a dust and atmospheric contamination larger than $7.8 \%(90 \% \mathrm{CL})$. Also the non-linear coupling parameter $f_{\mathrm{nl}}$ can be constrained to be $f_{\mathrm{nl}}=200_{-800}^{+1100}$ at the $95 \% \mathrm{CL}$ and on angular scales of 1.8 degrees. For comparison, the same method was applied to data from the NASA WMAP satellite in the same region of sky. The 1-year and 3-year releases were used. Results are compatible with those obtained with Archeops, implying in particular an upper limit for $f_{\mathrm{nl}}$ on degree angular scales.
\end{abstract}

Key words. cosmology: observations - methods: data analysis - cosmic microwave background

\section{Introduction}

According to the inflationary universe theory (e.g. Guth 1981; Linde 1990; Lyth \& Riotto 1998; Liddle \& Lyth 2000), the primordial density fluctuations are distributed following very precisely a Gaussian probability density function (pdf). These fluctuations in the matter density will produce anisotropies in the temperature of the Cosmic Microwave Background (CMB) whose pdf is also Gaussian. In this manner, when the Gaussianity of the CMB radiation is analysed the standard inflationary theory is tested as well as its alternatives (for example cosmic strings) which generically predict deviations from it in different ways. In addition, the search for non-Gaussianities has become a powerful tool to detect the presence of residual foregrounds, secondary anisotropies (such as gravitational lensing, Sunyaev-Zel'dovich effect) and unidentified systematic errors, which leave clearly non-Gaussian imprints on the CMB-anisotropies data.

There are many techniques to test Gaussianity, many of them developed previously as general statistical methods to test the normality of a data set, and others specifically for the CMB anisotropies. Among those methods, we can mention the estimator for non-Gaussianity based on the CMB bispectrum (Ferreira et al. 1998; Magueijo 2000), geometrical estimators on the sphere (Barreiro et al. 2001; Monteserín et al. 2005, 2006) Minkowski functionals(Gott et al. 1990; Komatsu et al. 2003), goodness-of-fit tests (Rayner \& Best 1989; Aliaga et al. 2003; Barreiro et al. 2007), wavelets (Ferreira et al. 1997;
Hobson et al. 1999; Barreiro et al. 2000) and steerable filters to search alignment structures (Wiaux et al. 2006).

Some of them have been applied to the CMB giving different results. For example WMAP data are compatible with Gaussianity according to the WMAP team (Komatsu et al. 2003; Spergel et al. 2007) whereas others have found evidence of nonGausssianities in the same WMAP maps, such as Copi et al. $(2004,2006)$ (using a technique called multipole vector framework), Eriksen et al. (2004, 2005) (finding asymmetries using local estimators of the n-point correlations), Vielva et al. (2004) and Cruz et al. (2005, 2006, 2007) (the Cold Spot detected with wavelets), Larson et al. (2004) (cold and hot spots different from those expected in Gaussian temperature fluctuations), among others.

In this study the smooth goodness-of-fit test first proposed by Rayner \& Best (1989, hereafter R\&BT) will be implemented to analyse the Gaussianity of the Archeops data. This method has already been applied successfully to the MAXIMA (Cayón et al. 2003b) and VSA experiments (Aliaga et al. 2005; Rubiño-Martín et al. 2006). The Archeops data will also be analysed with the morphological descriptors known as Minkowski functionals (Schmalzing \& Górski 1998; Gott et al. 1990). Our approach is to use both methods in the Gaussianity analysis for comparison of the sensitivities of the two techniques and crosschecking of the results on the amount of dust contamination and the amplitude of the non-linear coupling parameter.

This is the first Gaussianity analysis of the Archeops experiment data. We analysed the data for one of the Archeops bolometers at $143 \mathrm{GHz}$. This bolometer is the most sensitive and one of 
the most relevant for $\mathrm{CMB}$ observations. As a complementary analysis, we present the results of the same goodness-of-fit test applied to WMAP data with approximately the same mask as that used for Archeops to check whether the results are consistent for both data sets.

This paper is presented as follows: in Sect. 2 the R\&BT applied to signal-to-noise eigenmodes and the Minkowski functionals are described. The experiment, main properties of data sets and masks are summarised in Sect. 3. Section 4 is dedicated to the calibration and checking of both methods with some "realistic" CMB anisotropy Gaussian simulations, where we know in advance the output of the techniques. Section 5 contains the Archeops data analysis as well as results. In Sect. 6, WMAP 1-year and 3-year data are analysed and compared with Archeops results. Finally in Sect. 7 the main conclusions are presented.

\section{Goodness-of-fit tests and Minkowski functionals}

In this section, on the one hand we describe briefly the "goodness-of-fit technique" applied to test the Gaussianity of a set of signal-to-noise eigenmodes derived from measurements of the CMB temperature anisotropies. On the other hand, we will explain the Gaussianity analysis based on the Minkowski functionals.

\subsection{Smooth tests of goodness-of-fit}

Given a set of $n$ random numbers, $\left\{y_{i}\right\}_{i=1}^{i=n}$, it is sometimes interesting to check whether they behave statistically according to one specific pdf, $f(y, \theta)$, that is, if the probability of finding a random number $y$ in an interval between $y_{0}$ and $y_{0}+\Delta y$, with $\Delta y \geq 0$, is given by $f\left(y_{0}, \theta\right) \Delta y$. A scalar or vector variable $\theta$ is introduced, which allows us to move smoothly between different pdfs in their corresponding space of normalised functions.

This statistical analysis tests the null hypothesis, $H_{0}:\{\theta=0\}$ against the alternative hypothesis, $K:\{\theta \neq 0\}$.

From the family of smooth goodness-of-fit tests, we can consider an order $k$ alternative pdf $g_{k}(y, \theta)$, characterised by a pdf of the form (Rayner \& Best 1989, 1990)

$g_{k}(y, \theta)=C(\theta) \exp \left[\sum_{i=1}^{k} \theta_{i} h_{i}(y)\right] f(y)$

$\theta$ is a set of $k$ parameters to smoothly cover our space of pdfs, $f(y)$ is the null hypothesis pdf (e.g. the Gaussian distribution), $h_{i}(y)$ forms a complete set of orthonormal functions ${ }^{1}$ on $f(y)$, and $C(\theta)$ is a normalisation constant.

The "score statistic" is used to evaluate the simple null hypothesis $H_{0}$. With this statistic one can estimate the statistical significance of $\theta$ through the "Maximum Likelihood Method". Following the notation by Aliaga et al. (2003), the score statistic for this goodness-of-fit test is

$S_{k}=\sum_{i=1}^{k} U_{i}^{2}$,

and the $U_{i}^{2}$ quantities are given by

$U_{i}=\sum_{j=1}^{n} \frac{h_{i}\left(y_{j}\right)}{\sqrt{n}}$.

In the case of a Gaussian pdf, $h_{i}(x)$ are the "normalised HermiteChebyshev polynomials". If the null hypothesis is satisfied then

\footnotetext{
${ }^{1} \int_{-\infty}^{\infty} h_{i}(y) f(y) h_{j}(y) \mathrm{d} y=\delta_{i j}$.
}

the $U_{i}$ quantities have statistically normal behaviour and therefore $U_{i}^{2}$ behave like a $\chi_{1}^{2}$ distribution

$f\left(U_{i}^{2}\right)=\frac{1}{\sqrt{2 \pi U_{i}^{2}}} \mathrm{e}^{-\frac{-U_{i}^{2}}{2}}$.

It is possible to write the $U_{i}^{2}$ statistical quantities in terms of the moments of order $k$ derived from the set of $n$ random numbers to be analysed, $\mu_{k}=1 / n \sum_{j=1}^{n} y_{j}^{k}$, (e.g. Aliaga et al. 2003, 2005).

We used the five first statistics $U_{i}^{2}$ which can be related to the $k$-order moments as follows,

$U_{1}^{2}=n\left(\mu_{1}\right)^{2}$

$U_{2}^{2}=\frac{n}{2}\left(\mu_{2}-1\right)^{2}$

$U_{3}^{2}=\frac{n}{6}\left(\mu_{3}-3 \mu_{1}\right)^{2}$

$U_{4}^{2}=\frac{n}{24}\left(\mu_{4}-6 \mu_{2}+3\right)^{2}$

$U_{5}^{2}=\frac{n}{120}\left(\mu_{5}-10 \mu_{3}+15 \mu_{1}\right)^{2}$.

The first few statistics are generally the most sensitive for most applications. In our case higher order $U_{i}^{2}$ statistics are dominated by errors (because of the usual propagation of errors) and therefore are not very useful in practice. This is described in detail in Sect. 4.

\subsection{Signal-to-noise eigenmode analysis}

We have described the method that is used to analyse a set of $n$ random numbers to test whether their pdf is the normal distribution.

The next step is to compute the set of numbers to be analysed. In our case they come from the so-called "signal-to-noise eigenmodes", first introduced in the CMB field by Bond (1995). Our observational data, (the fluctuation in the temperature of the incoming blackbody radiation measured for each direction $\boldsymbol{n}$ in the sky, $\Delta T(\boldsymbol{n}) / T$ ), can be interpreted as originating from several sources: all emissions coming from the sky (CMB signal, Galactic and extragalactic foregrounds and atmosphere) and the measured instrumental Gaussian noise (Macías-Pérez et al. 2007).

The total area observed by the experiment is usually divided into equal area pixels identified by their centre direction $\boldsymbol{n}$ and to which the measurements, $\Delta T(\boldsymbol{n}) / T$, are assigned. To obtain the signal-to-noise eigenmodes, we expand the pixel values of the map, $\Delta T(\boldsymbol{n}) / T$, into a linear combination in which the transformed instrumental noise (hereafter the noise) and the transformed theoretical CMB signal (hereafter the signal) are not correlated.

For the signal-to-noise decomposition it is necessary to calculate signal and noise covariance matrices. The temperature covariance between two pixels $i$ and $j$ is given by

$C_{i j}=\left\langle\Delta T_{i} \Delta T_{j}\right\rangle-\left\langle\Delta T_{i}\right\rangle\left\langle\Delta T_{j}\right\rangle$

where the brackets \langle\rangle represent the average over several realisations of temperature anisotropy maps. Thus we can construct the signal (noise) covariance matrices, $S(N)$, averaging over signal $\Delta T_{\mathrm{s}}(\boldsymbol{n})$ (noise $\Delta T_{\mathrm{n}}(\boldsymbol{n})$ ) realisations. Since the data represent temperature fluctuations around the mean then it is trivially satisfied that $\left\langle\Delta T_{\mathrm{s}}(\boldsymbol{n})\right\rangle=\left\langle\Delta T_{\mathrm{n}}(\boldsymbol{n})\right\rangle=0$. Therefore, $C_{i j}=\left\langle\Delta T_{i} \Delta T_{j}\right\rangle$, the correlation matrix. 
Once we select a set of $n$ directions in the sky (pixels) and construct $S$ and $N$ matrices, which have the same dimension $n$ and are symmetrical, we can compute the "signal-to-noise matrix" A

$A=L_{N}^{-1} S L_{N}^{-t}$

where $L_{N}$ is the Cholesky matrix of $N$, defined as $N \equiv L_{N} L_{N}^{t}$. $L_{N}$ can be obtained from the diagonalisation of the $N$ matrix. Suppose $D_{N}$ is the diagonal matrix of eigenvalues of $N$, and $R_{N}$ is a matrix of the eigenvectors of $N$, related by $R_{N}^{t} N R_{N}=D_{N}$, then $L_{N}=R_{N} D_{N}^{1 / 2}$ is satisfied where $D_{N}^{1 / 2}$ is the square root matrix of $D_{N}$.

If $\boldsymbol{d}$ is the vector of dimension $n$ representing the data assigned to the pixels in the sky, the signal-to-noise eigenmodes can be written as

$\boldsymbol{\xi}=R_{A}^{t} L_{N}^{-1} \boldsymbol{d}$

where $R_{A}$ is the matrix of eigenvectors of $A$ and $D_{A}$ the diagonal matrix of eigenvalues of $A, R_{A}^{t} A R_{A}=D_{A}$.

The $y_{i}$ quantities to be analysed with the goodness-of-fit test defined in the previous section are

$y_{i}=\frac{\xi_{i}}{\sqrt{1+\left(D_{A}\right)_{i}}}$.

It can be easily demonstrated that if the vector of data $\boldsymbol{d}$ satisfies $\langle\boldsymbol{d}\rangle=0$ then $\left\langle y_{i}\right\rangle=0$. In the case $\Delta T=\Delta T_{\mathrm{s}}+\Delta T_{\mathrm{n}}$, from the definition of signal-to-noise eigenmodes in Eq. (8), the definition of $y_{i}$ in Eq. (9), and properties of correlation matrices, it follows that $\left\langle y_{i}^{2}\right\rangle=1$.

Supposing that the original map $\boldsymbol{d}$ is multi-normal, then our $\left\{y_{i}\right\}$ numbers keep the Gaussian character because both sets of numbers are connected by linear operations. More precisely, they follow a normal pdf with zero mean and unit variance, $N(0,1)$. Moreover, for different indexes $i$ and $j, y_{i}$ and $y_{j}$ are independent.

Finally, for Gaussian data $\boldsymbol{d}$ each $U_{i}^{2}$ statistics, defined in Eq. (3), is distributed as a $\chi_{1}^{2}$. The decision to accept or reject the null hypothesis will therefore be based on this pdf, as is seen in Sects. 5 and 6 when the test is applied to the Archeops and WMAP data.

\subsection{Minkowski functionals}

Considering the temperature anisotropies of the CMB as a scalar field on the sphere we can define the set of coordinates $Q_{v}$ where $\Delta T(\boldsymbol{n})>v$ for a given threshold $v$, and its complementary set $V_{v}$. As stated in Schmalzing \& Górski (1998), any morphological descriptor on the sphere is a linear combination of 3 Minkowski functionals. These functionals are: the area $A(v)$ of the excursion set $Q_{v}$, the contour length $C(v)$ of the excursion set $Q_{v}$, and the genus $G(v)$ (defined as the number of hot spots above $v$ minus the number of cold spots below that threshold).

For a Gaussian random field, the mean values of these functionals are

$$
\begin{aligned}
& \langle A(v)\rangle=\frac{1}{2}\left(1-\frac{2}{\sqrt{\pi}} \int_{0}^{v / 2} \exp \left(-t^{2}\right) \mathrm{d} t\right) \\
& \langle C(v)\rangle=\frac{\sqrt{\tau}}{8} \exp \left(-\frac{v^{2}}{2}\right) \\
& \langle G(v)\rangle=\frac{\tau}{(2 \pi)^{3 / 2}} v \exp \left(-\frac{v^{2}}{2}\right)
\end{aligned}
$$

where $\tau$ is a parameter related with the coherence angle (Barreiro et al. 2001; Schmalzing \& Górski 1998).

The Gaussianity test with the Minkowski functionals is performed through a $\chi^{2}$ test as described for example in Komatsu et al. (2003) and Spergel et al. (2007) for WMAP data, and de Troia et al. (2007) for BOOMERanG 2003 data. Considering $n_{\text {th }}$ possible thresholds $v$, we can define a $3 n_{\text {th }}$ vector $v=(A(v), C(v), G(v))$. The $\chi^{2}$ statistic is then defined

$\chi^{2}=\sum_{i, j}(\boldsymbol{v}(i)-\langle\boldsymbol{v}(i)\rangle) C_{i j}^{-1}(\boldsymbol{v}(j)-\langle\boldsymbol{v}(j)\rangle)$

where $\langle\boldsymbol{v}(j)\rangle$ is the expected value of $v(j)$ and $C$ is the corresponding covariance matrix for all possible thresholds and functionals.

\section{Archeops data sets}

\subsection{The Archeops experiment}

Archeops $^{2}$ is a balloon-borne experiment dedicated to measure the CMB temperature anisotropies from large to small angular scales (Benoît et al. 2003a; Tristram et al. 2005). It has given the first link in the $C_{\ell}$ determination between the COBE large angular scales data (Smoot et al. 1992) to the first acoustic peak as measured by BOOMERanG and MAXIMA (de Bernardis et al. 2000; Hanany et al. 2000). Archeops was also designed as a testbed for the forthcoming Planck High Frequency Instrument (HFI), (Lamarre et al. 2003). Therefore, Archeops shared with Planck the same technological design: a Gregorian off-axis telescope with a $1.5 \mathrm{~m}$ primary mirror, bolometers operating at 143 , 217,353 and $545 \mathrm{GHz}$ cooled down at $100 \mathrm{mK}$ by a ${ }^{3} \mathrm{He} /{ }^{4} \mathrm{He}$ dilution designed to work at zero gravity and a similar scanning strategy. Archeops was launched on February 7th, 2002, from the CNES/Swedish facility of Esrange, near Kiruna (Sweden). Twelve hours of high quality night data were gathered. This data corresponds to a coverage of approximately $30 \%$ of the sky, including the Galactic plane. More details about the instrument and the flight performance can be found in Benoît et al. (2003b) and Macías-Pérez et al. (2007). From its four frequency bands the two lowest (143 and $217 \mathrm{GHz}$ ) were dedicated to the observation of the $\mathrm{CMB}$ and the others $(353$ and $545 \mathrm{GHz}$ ) to the monitoring and calibration of both atmospheric and Galactic emissions.

In the following, we focus on the analysis of the most sensitive $143 \mathrm{GHz}$ Archeops bolometer which also presents the lowest level of contamination by systematic effects.

Although the Archeops resolution is typically 10 arcmin, for this analysis we are interested in the Gaussianity of the large angular scale anisotropies. Therefore, we decided to use low resolution maps at HEALPix (Górski et al. 2005) $N_{\text {side }}=32$ to consider scales above 1.8 degrees.

\subsection{Data processing}

We describe here briefly the way that Archeops data were processed. For a more detailed description see Macías-Pérez et al. (2007).

In the Time Ordered Information (TOI) corrupted data are flagged (representing less than $1.5 \%$ of the whole data set). Low frequency drifts, correlated to house-keeping data are removed using the latter as templates. A high frequency decorrelation is

\footnotetext{
${ }^{2}$ http://www.archeops.org
} 


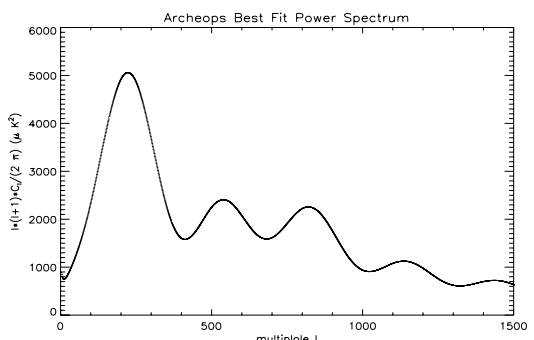

Fig. 1. Archeops Best Fit Power Spectrum used to simulate the Archeops CMB signal.

also performed to remove some bursts of non-stationary highfrequency noise. Corrected timelines are then deconvolved from the bolometer time constant and the flagged corrupted data are replaced by a realisation of noise. Finally, low time frequency atmospheric residuals are subtracted using a destriping procedure which slightly filters out the sky signal to a maximum of $5 \%$.

Archeops-cleaned TOIs at $143 \mathrm{GHz}$ are contaminated by atmospheric and Galactic dust residuals, even at intermediate Galactic latitudes. Atmospheric residuals contribute mainly at frequencies lower than $2 \mathrm{~Hz}$ in the timeline and follow approximately a $v^{2}$ law in antenna temperature. Galactic dust presents a grey body spectrum at about $17 \mathrm{~K}$ with an emissivity of about $v^{2}$. To suppress both residual dust and atmospheric signals, data are decorrelated using a linear combination of the high frequency photometric pixels (353 and $545 \mathrm{GHz}$ ) and of synthetic dust timelines.

In this study we used two kinds of map-making for the TOIs of Archeops data and of the simulations. The first one is an optimal map-making procedure called Mirage (Yvon \& Mayet 2005). Mirage is based on a two-phase iterative algorithm, involving optimal map-making together with low frequency drift removal and Butterworth high-pass filtering. A conjugate gradient method is used for resolving the linear system. The second is a procedure that performs coaddition. This means that all TOI points corresponding to a given pixel are averaged.

To produce a CMB simulation, a random CMB map with the power spectrum of the Archeops model (Benoît et al. 2003b, Fig. 1) is generated and from this map an Archeops TOI is produced. This TOI is treated with the two map-making methods described above to produce a map. To perform a noise simulation we produce a Gaussian constrained realisation of the Archeops noise power spectrum in the time domain. The TOI produced this way is then projected onto a map using the above map-making techniques.

The analysis was performed on a fraction of the Archeops observed region masking out pixels with Galactic latitude below 15 degrees, $|b|<15^{\circ}$. The southern sky data were not included in the analysis as they are more contaminated by systematics in the form of residual stripes coming from the Fourier filtering and destriping of the data in the time domain (Macías-Pérez et al. 2007) which produces ringing around the Galactic plane. The southern sky region was used in the CMB power spectrum analysis (Tristram et al. 2005) because it increased significantly the signal-to-noise ratio at small angular scales. These scales are not affected by this systematic effect. This is not the case for the analysis presented in this paper where we are more interested in large angular scales where this systematic becomes important. In Fig. 2 we plot the region of data considered for the analysis. These data correspond to 1995 pixels ( $16 \%$ of the sky) from a total of 12288 pixels for a complete map at this resolution.

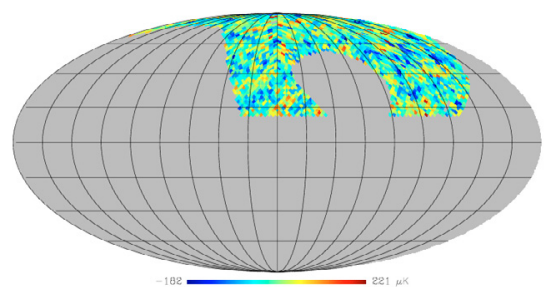

Fig. 2. Mirage Archeops data from the best bolometer at $143 \mathrm{GHz}$ presented at HEALPix resolution $N_{\text {side }}=32,(\approx 1.8$ degrees $)$. This map is centred on Galactic longitude $l=180$ degrees. Galactic and South Equator pixels have been masked. Grid lines are spaced by 20 degrees.

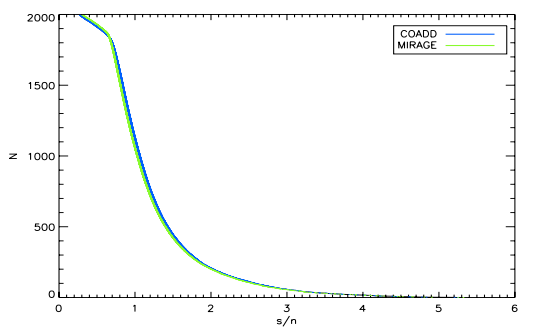

Fig. 3. Number of normalised signal-to-noise eigenmodes $y_{i}$ for which their associated $A$ matrix eigenvalues, $\left(D_{A}\right)_{i}$, satisfy $\left(D_{A}\right)_{i} \geq(s / n)_{\mathrm{c}}^{2}$.

\section{Calibrating the method: analysis on Gaussian simulations}

To develop the R\&BT non-Gaussianity test, it is necessary to calculate the signal $(\mathrm{S})$ and the noise $(\mathrm{N})$ correlation matrices among the selected pixels. We computed these matrices averaging simulations by means of Eq. (6). For this purpose Monte Carlo Gaussian simulations of Archeops CMB signal and instrumental noise were produced. The number of simulations performed for the map generated with the Mirage map-making procedure were $2.86 \times 10^{5}$ for the signal and $2.75 \times 10^{5}$ for the noise, whereas for the coaddition procedure they were $5 \times 10^{5}$ and $5 \times 10^{5}$ for the signal and noise respectively. Ninety dualcore $3.2 \mathrm{GHz}$ processors from the IFCA computing facilities were used. Each Mirage simulation took 180 s of real CPU time and 1.0 GB of RAM memory, whereas these values were $70 \mathrm{~s}$ and $0.04 \mathrm{~GB}$ respectively for each coaddition simulation.

The high number of simulations and the corresponding computational requirements were needed to achieve convergence in the construction of the correlation matrices. The main reason for the low convergence relies on the specific properties of our correlation matrices. Archeops noise is correlated at large scales, which means that the $N$ matrix is neither diagonal nor sparse. The Archeops signal correlation matrix contains correlations at large scales for which the convergence is much slower than for the small scales due to the cosmic variance. In both cases many simulations $\left(\sim 10^{5}\right)$ were required to compute these matrices.

One way to quantify the degree of convergence of these matrices is by analysing Gaussian simulations. The $U_{i}^{2}$ statistics for a set of Gaussian simulations should have a $\chi_{1}^{2}$ pdf. This can be tested, for example, by calculating the mean and the variance of the $U_{i}^{2}$ statistics for $10^{4}$ Gaussian signal plus noise simulations. For the Gaussian case, the mean should be equal to 1 and the dispersion equal to $\sqrt{2}$ (this is the null hypothesis, $H_{0}$ ).

Following Aliaga et al. (2005) and Rubiño-Martín et al. (2006), the $U_{i}^{2}$ are computed for a subset of signal-to-noise eigenmodes which are those associated with eigenvalues of the signal-to-noise matrix $A$ satisfying $\left(D_{A}\right)_{i} \geq(s / n)_{\mathrm{c}}^{2}$, where $(s / n)_{\mathrm{c}}$ is a given signal-to-noise ratio cut. In Fig. 3 the number of 

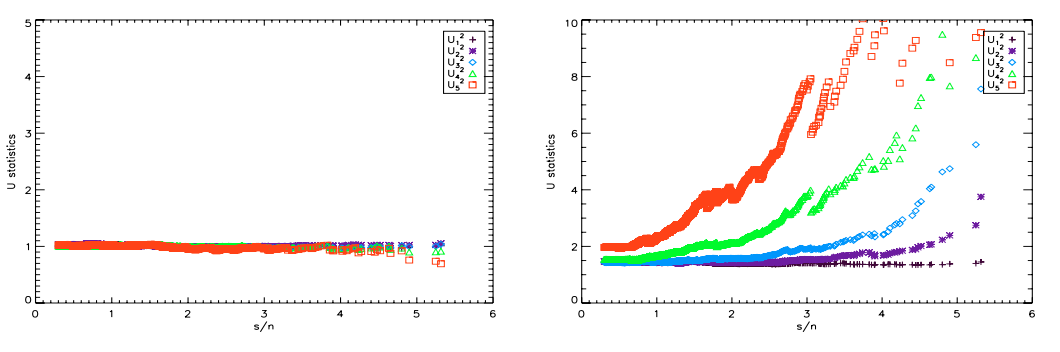

Fig. 4. From left to right, mean and dispersion of $U_{i}^{2}$ statistics (where $i$ goes from 1 to 5) for different signal-to-noise cuts, corresponding to $10^{4}$ signal plus noise Mirage simulations.
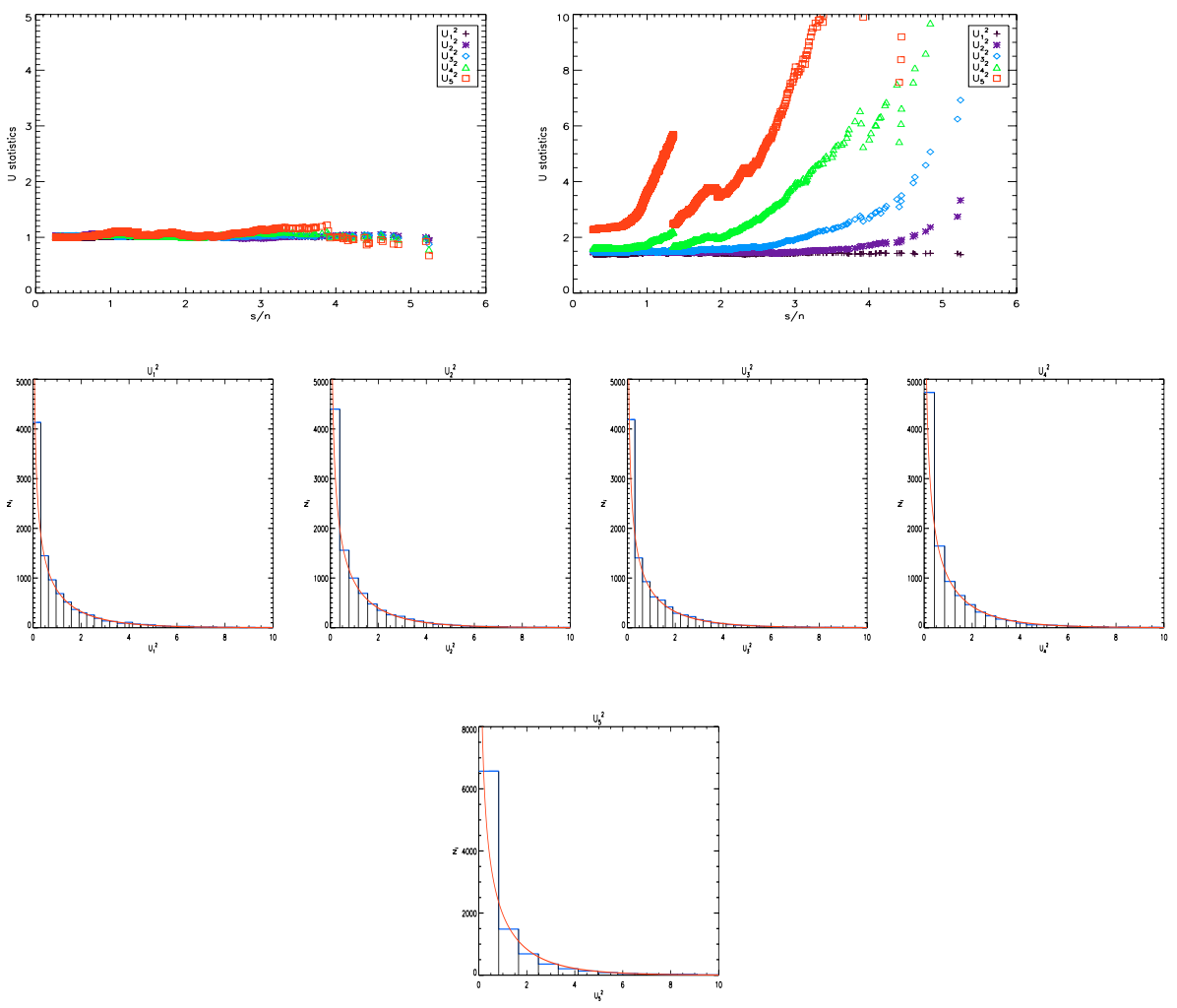

eigenmodes $\left\{y_{i}\right\}$ 's, which obey $\left(D_{A}\right)_{i} \geq(s / n)_{\mathrm{c}}^{2}$, in terms of $s / n$ is plotted.

In Fig. 4 we show the mean and dispersion of the five first $U_{i}^{2}$ statistics for different signal-to-noise cuts corresponding to all possible eigenvalues of the $A$ matrix. The $U_{i}^{2}$ values come from a set of $10^{4}$ Gaussian Archeops signal plus noise Mirage simulations. It can be seen that for $(s / n)_{\mathrm{c}} \lesssim 2$ mean values are close to 1 and the dispersion close to $\sqrt{2}$ (except for the $U_{5}^{2}$ statistic whose dispersion is always larger than 2). As shown by e.g. Aliaga et al. (2005), the expected value of $U_{i}^{2}$ is equal to 1 independent of the number of $\left\{y_{i}\right\}$ used. This explains why the mean of $U_{i}^{2}$ is very close to 1 for every signal-to-noise cut. The dispersion is equal to $\sqrt{2}$ asymptotically, when the number of $\left\{y_{i}\right\}$ used is high. In our case, this happens for low signal-to-noise cuts, when enough $\left\{y_{i}\right\}$ s are used to compute the statistics. In Fig. 5 the same quantities have been plotted for the $10^{4}$ Gaussian Archeops signal plus noise coaddition simulations. Similar conclusions can be derived in this case. Notice, however, that the results are closer to theoretical values when the analysis is performed using the Mirage maps. In this case, the correlation matrices have converged with fewer simulations than in the coaddition case. This is one of the advantages of using Mirage simulations over the coaddition ones, although the production of a Mirage map requires more CPU time and RAM memory than a coaddition map.
Since the computation of high order $U_{i}^{2}$ statistics involves high powers of the eigenmodes, the convergence of their dispersion to the theoretical values at a given $(s / n)_{\mathrm{c}}$ is slower than for the low order ones (as seen in the right panels of Figs. 4 and 5).

A more exhaustive check for the convergence of the $U_{i}^{2}$ statistics is done by comparing their theoretical pdf with the histograms obtained from the simulated data. Given a signal-tonoise ratio cut $(s / n)_{\mathrm{c}}$ for the calculation of the $U_{i}^{2}$ statistics, it is possible to make a histogram with the corresponding values of the $U_{i}^{2}$ statistics from the same sets of $10^{4}$ simulations. Figure 6 compares the histograms for the first five statistics calculated using all the eigenmodes $(s / n \geq 0.30)$ for the Mirage simulations with the theoretical expectation of a $\chi_{1}^{2}$ distribution. In Table 1 the mean and the dispersion of these histograms are presented. In Fig. 7 the same comparison is shown for the coaddition simulations also considering all the eigenmodes $(s / n \geq 0.27)$. The corresponding mean and dispersion of these histograms are given in Table 2.

In summary, the four statistics $U_{1}^{2}, U_{2}^{2}, U_{3}^{2}$, and $U_{4}^{2}$ have pdfs compatible with the theoretical one whereas $U_{5}^{2}$ starts to deviate from it. The discrepancy, already present in the dispersion, increases for higher orders. The reason is that high order moments enlarge possible errors present in the computed correlation matrices and are propagated in the diagonalisation processes. In any case, the $U_{5}^{2}$ statistic can still be used for the Gaussian analysis if the distribution obtained from the simulations, instead of 

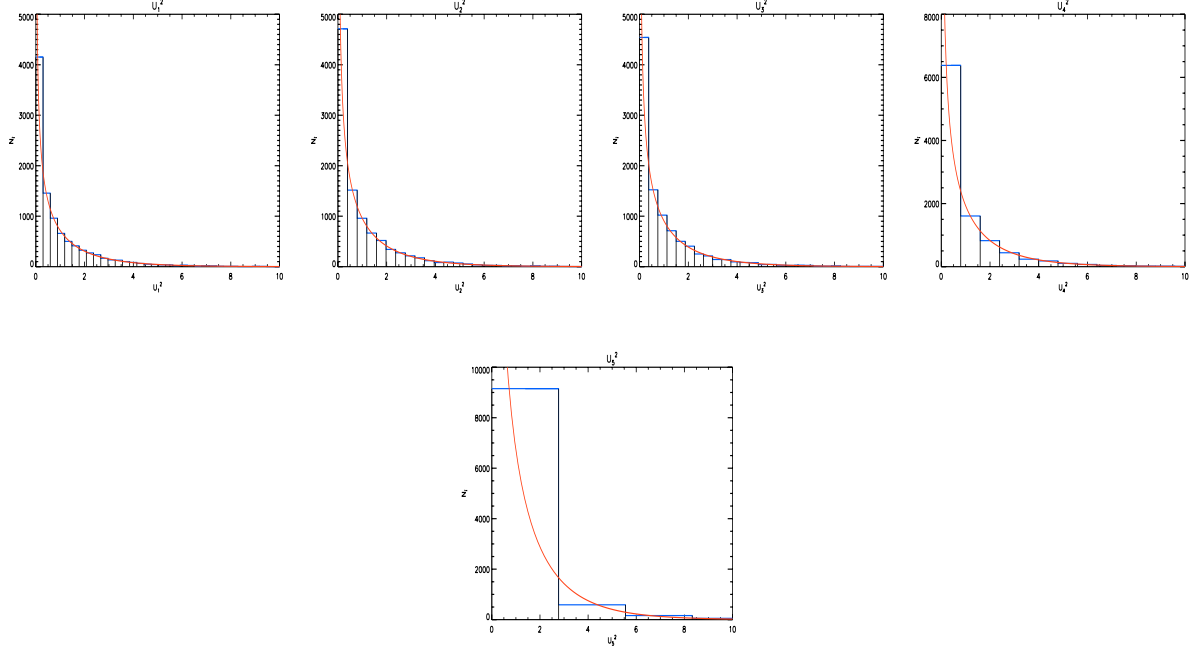

Fig. 7. From left to right, and from top to bottom, distribution of the $U_{i}^{2}$ statistics, from a set of $10^{4}$ Gaussian coaddition simulations analysed in the same region as the data (Fig. 2). The signal-to-noise cut which has been used is $(s / n)_{\mathrm{c}}=0.27$. Solid lines are the theoretical distribution $\left(\chi_{1}^{2}\right)$ normalised to the number of simulations and the size of the binned cell.
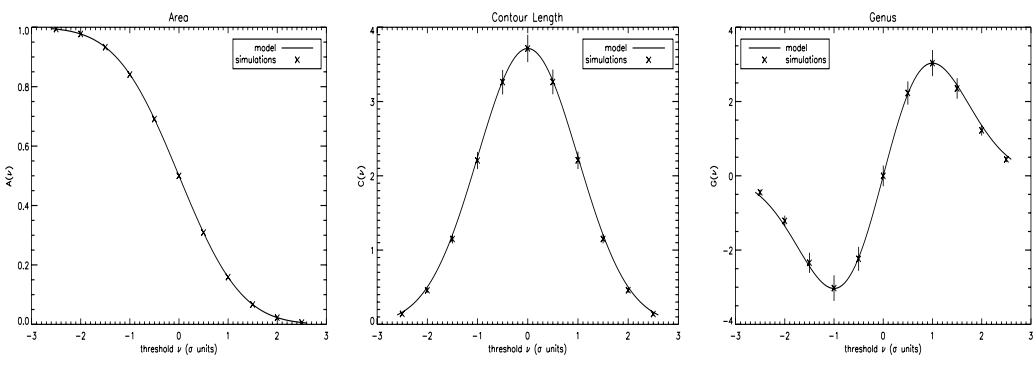

Fig. 8. From left to right, mean values of the three Minkowski functionals and their corresponding error-bars for a set of 1000 (noiseless) CMB Gaussian simulations. These simulations have been generated using Archeops best fit power spectrum and have not been masked. Note the good agreement between the theoretical predictions and the results obtained from simulations.

Table 1. Mean and dispersion of $U_{i}^{2}$ statistics from $10^{4}$ Mirage simulations for a signal-to-noise ratio cut of 0.30 .

\begin{tabular}{c|ccccc|c}
\hline \hline$\ldots$ & $U_{1}^{2}$ & $U_{2}^{2}$ & $U_{3}^{2}$ & $U_{4}^{2}$ & $U_{5}^{2}$ & $\chi_{1}^{2}$ \\
\hline$\mu$ & 1.02 & 1.04 & 1.01 & 1.01 & 1.02 & 1.00 \\
$\sigma$ & 1.45 & 1.47 & 1.43 & 1.55 & 1.96 & 1.41 \\
\hline
\end{tabular}

Table 2. Mean and dispersion of $U_{i}^{2}$ statistics from $10^{4}$ coaddition simulations for a signal-to-noise ratio cut of 0.27 .

\begin{tabular}{c|ccccc|c}
\hline \hline$\ldots$ & $U_{1}^{2}$ & $U_{2}^{2}$ & $U_{3}^{2}$ & $U_{4}^{2}$ & $U_{5}^{2}$ & $\chi_{1}^{2}$ \\
\hline$\mu$ & 0.99 & 1.02 & 1.02 & 1.02 & 1.00 & 1.00 \\
$\sigma$ & 1.40 & 1.47 & 1.48 & 1.62 & 2.27 & 1.41 \\
\hline
\end{tabular}

the theoretical one, is used as reference. Although this is not as optimal as using the theoretical $\chi_{1}^{2}$ distribution, it is however a good compromise taking into account the huge computational resources needed to produce a very large number of simulations.

For the Minkowski functionals analysis the expected values given by Eq. (10) cannot be applied to our problem because of the contour restrictions of the mask and the presence of anisotropic noise. Nevertheless in order to test our Minkowski functional codes we performed an analysis on (noiseless) CMB Gaussian simulations over all sky and 1.8 degrees resolution generated using the best fit Archeops power spectrum. Analysing them for thresholds from $-2.5 \sigma$ to $2.5 \sigma$ (where $\sigma$ is the standard deviation of the corresponding simulation), we obtained results from simulations compatible with the theoretical predictions (Fig. 8).

\section{Gaussianity test on Archeops data}

We applied the R\&BT to the Archeops 143 K03 bolometer map. The signal-to-noise eigenmodes were computed with the correlation matrices described in Sect. 4, for each map-making case. We checked in that section that these signal and noise matrices provide $U_{i}^{2}$ statistics compatible with Gaussianity for Gaussian simulations.

We applied this test to the Archeops data for the Mirage and coaddition map-making. The $U_{i}^{2}$ statistics, computed for the 1995 pixels of the previously described Archeops data, are displayed in Figs. 9 and 10. The $U_{i}^{2}$ statistics are plotted, from $i=1$ to 5 , versus the signal-to-noise eigenmode cut.

For the Mirage map-making, results are displayed in Fig. 9. We can see that all the $U_{i}^{2}$ statistics are below 5 for all the signal-to-noise cuts. This means that the data are compatible with Gaussianity.

For coaddition map-making, we can see from Fig. 10 that whatever the signal-to-noise eigenmode cut is, $U_{i}^{2}$ statistics for the $143 \mathrm{~K} 03$ bolometer data are below 5, except for $U_{2}^{2}$ for signalto-noise cuts below 0.5 . It reaches the maximum value of 7.97 at the minimum signal-to-noise cut of 0.27 . The upper tail probability $^{3}$ for $U_{2}^{2}=7.97$ from the $\chi_{1}^{2}$ distribution (Eq. (4)) is $0.5 \%$. Comparing with the set of coaddition Gausian simulations we found that this upper tail probability is $0.6 \%$, (Table 3 ), in good agreement with the theoretical expectation. Nevertheless, as we computed $U_{i}^{2}$ statistics for all possible signal-to-noise cuts, it is important to estimate the significance of finding any simulation with $U_{2}^{2} \geq 7.97$ in at least one of them. This is the so-called " $p$-value" of $U_{2}^{2}$. The " $p$-value" is defined as the probability that the relevant statistic takes a value at least as extreme as that observed in the data when the null hypothesis is true. We found for $U_{2}^{2}$ that the " $p$-value" is $15.0 \%$. 


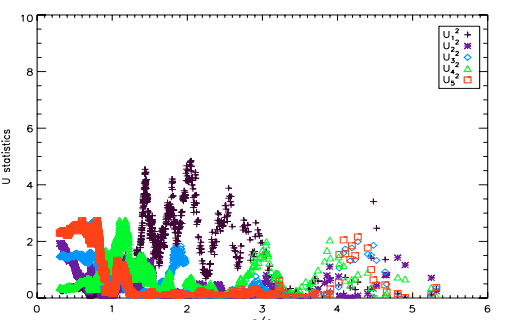

Fig. 9. $U_{i}^{2}$ statistics of Mirage Archeops Data for different signal-tonoise cuts.

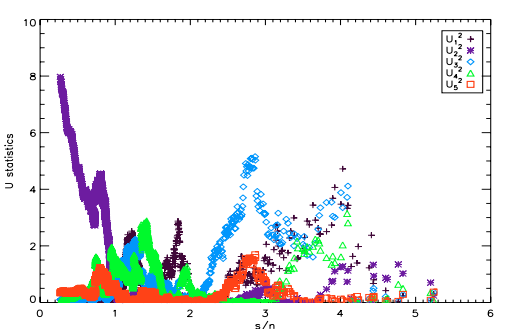

Fig. 10. $U_{i}^{2}$ statistics of coaddition Archeops Data for different signalto-noise cuts.

Table 3. $U_{i}^{2}$ from Archeops Mirage (coaddition) map for $(s / n)_{\mathrm{c}}=0.30$ $\left((s / n)_{\mathrm{c}}=0.27\right)$ and the probability that one $s+n$ Gaussian Mirage (coaddition) simulation has a $U_{i}^{2}$ statistic larger than those of the data. More precisely, the probability for $U_{2}^{2}$ in the coaddition case is $0.6 \%$.

\begin{tabular}{c|ccccc}
\hline \hline$\ldots$ & $U_{1}^{2}$ & $U_{2}^{2}$ & $U_{3}^{2}$ & $U_{4}^{2}$ & $U_{5}^{2}$ \\
\hline Mirage & 0.28 & 1.92 & 1.45 & 0.38 & 2.29 \\
Prob. & 0.60 & 0.17 & 0.23 & 0.54 & 0.12 \\
\hline Coaddition & 0.11 & 7.97 & 0.10 & 0.04 & 0.34 \\
Prob. & 0.73 & 0.01 & 0.75 & 0.83 & 0.52 \\
\hline
\end{tabular}

We can thus conclude that even if we have a relatively strong $U_{2}^{2}$ at the lowest signal-to-noise ratio, it is not improbable to have such a high value by chance. Therefore, even considering the results from the coaddition map-making, Archeops data is still compatible with our Gaussian simulations.

Although the high value found for $U_{2}^{2}$ for the coaddition map is not significant enough to be incompatible with Gaussianity, it is clear that there is a steady increase of $U_{2}^{2}$ when $s / n$ decreases. This suggests the presence of systematics in the coaddition maps which can depend on the resolution. Moreover, the fact that it only appears in coaddition data suggests the possibility that it is a map-making issue. This also implies that systematics are better controlled in the Mirage than in the coaddition map-making. Therefore hereafter we focus only on the Mirage map-making data.

We performed a $\chi^{2}$ test with the three Minkowski functionals using 11 thresholds from $-2.5 \sigma$ to $2.5 \sigma$. We analysed the Mirage data and a set of 1000 CMB Gaussian simulations with noise of the Mirage type. The corresponding histogram of the $\chi^{2}$ values of these simulations and of the data are presented in Fig. 11. As can be seen, the data are compatible with the Gaussian simulations.

\subsection{Systematic and foreground contamination}

The R\&BT can also provide a powerful tool for estimating the level of this contribution. The test consists of adding different percentages of a template map to the Archeops $143 \mathrm{~K} 03$

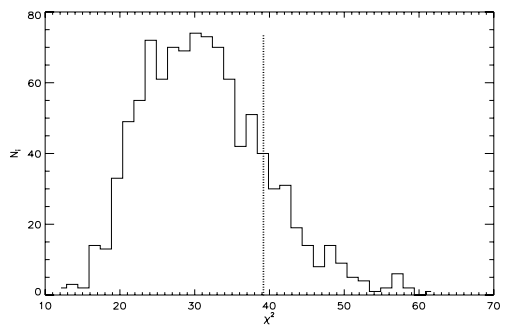

Fig. 11. Distribution of the $\chi^{2}$ values from the Minkonwski Gaussianity test for Archeops Mirage map. Vertical line shows the data results. Their cumulative probability is $83.9 \%$.

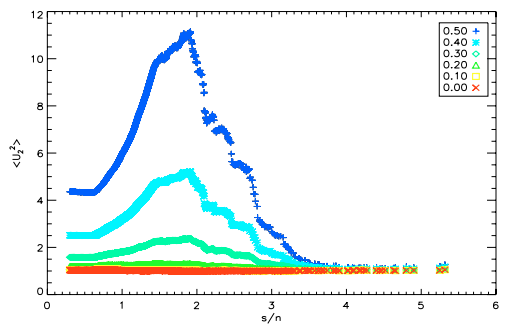

Fig. 12. Mean of $10^{4} U_{2}^{2}$ statistics, from $10^{4}$ signal plus noise Mirage simulations plus a factor $\alpha_{\mathrm{d}}$ times the contamination template. $0.0 \leq$ $\alpha_{\mathrm{d}} \leq 0.5$.

bolometer map, for the Mirage and coaddition simulations cases, to compare the resulting $U_{i}^{2}$ statistics to those obtained with the Archeops data at $143 \mathrm{GHz}$.

This template map is computed from the coadded Archeops $353 \mathrm{GHz}$ map (Ponthieu et al. 2005). This map contains thermal dust emission, atmospheric residuals as the dominant components and also instrumental noise and CMB residuals. Thus, extrapolated to $143 \mathrm{GHz}$ it will provide a good template of what could be a dust plus atmospheric contamination at this frequency.

Thermal dust is assumed to have a grey-body emission: $v^{2} B(v)$ which can be approximated in the Rayleigh-Jeans domain to $T_{\mathrm{RJ}} \propto v^{2}$ (Ponthieu et al. 2005). The atmospheric residuals emission law has been estimated empirically by the Archeops collaboration (Macías-Pérez et al. 2007) and is also proportional to $v^{2}$ in the Rayleigh-Jeans domain. Dust and atmospheric residuals being the two main components, the Archeops $353 \mathrm{GHz}$ map has been extrapolated to $143 \mathrm{GHz}$ by assuming this emission power law. Due to the extrapolation, the CMB contribution on the $353 \mathrm{GHz}$ template map is negligible with respect to the CMB at $143 \mathrm{GHz}$.

$U_{2}^{2}$ statistic is the most sensitive to this effect as can be seen in Fig. 12 for the Mirage case where this statistic presents a prominent peak at signal-to-noise ratio cuts around 1.88.

To determine the level of contamination we performed a $\chi^{2}$ test with the $U_{2}$ statistic computed at $(s / n)_{\mathrm{c}}=1.88$. It is optimal to perform a $\chi^{2}$ test with $U_{2}$ because $U_{2}$ is normally distributed for the null hypothesis. Thus we can define

$\chi^{2}\left(\alpha_{\mathrm{d}}\right)=\frac{1}{\sigma_{\alpha_{\mathrm{d}}}^{2}\left(U_{2}\right)}\left(U_{2}-\left\langle U_{2}\right\rangle_{\alpha_{\mathrm{d}}}\right)^{2}$

where $\left\langle U_{2}\right\rangle_{\alpha_{\mathrm{d}}}$ and $\sigma_{\alpha_{\mathrm{d}}}\left(U_{2}\right)$ are the mean and the dispersion of $U_{2}$ for CMB Gaussian simulations with noise plus a factor $\alpha_{\mathrm{d}}$ times the contamination template. In the left panel of Fig. 13 we present the $\chi^{2}$ of Archeops Mirage data for different $\alpha_{\mathrm{d}}$. We can see that the minimum $\chi^{2}$ (best fit) occurs for $\alpha_{\mathrm{d}}=0.0$. Analysing Gaussian simulations without dust we find that most of them reach the best fit for low values of $\alpha_{\mathrm{d}}$ (right panel; 

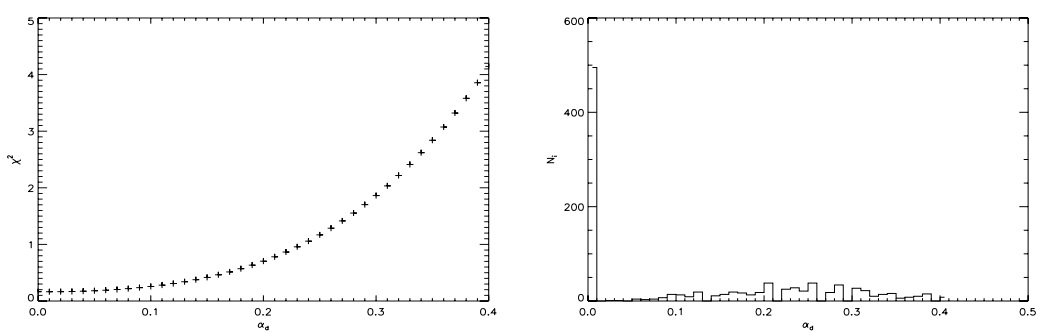

Fig. 13. From left to right, $\chi^{2}$ value of Archeops data for different $\alpha_{\mathrm{d}}$ and the histogram of best fit $\alpha_{\mathrm{d}}$ for a set of 1000 Gaussian simulations without dust. These results have been obtained with the $U_{2}$ statistic.
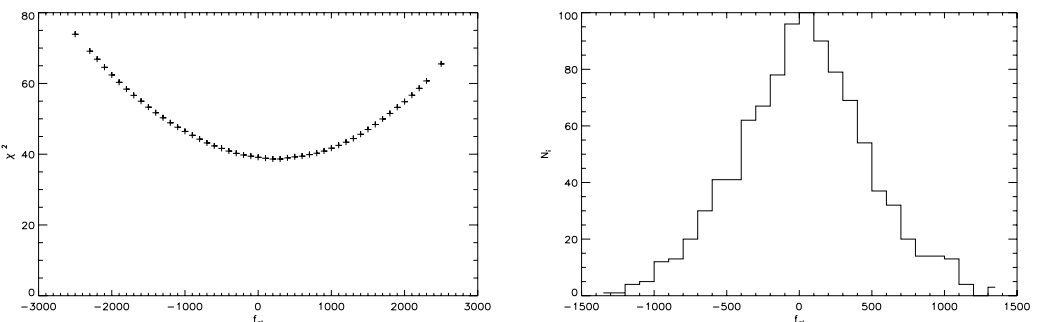

Fig. 14. From left to right, $\chi^{2}$ value of Archeops data for different $f_{\mathrm{nl}}$ and the histogram of best fit $f_{\mathrm{nl}}$ for a set of 1000 CMB Gaussian simulations with noise. Results were obtained with the Minkowski functionals.

Fig. 13). Specifically $\alpha_{\mathrm{d}} \leq 0.27$ for $90 \%$ confidence level (CL), and $\alpha_{\mathrm{d}} \leq 0.33$ for $95 \% \mathrm{CL}$. By comparing the dispersion of both maps, Archeops and 0.27 times the contamination template, we can exclude a dust plus atmospheric contamination larger than $7.8 \%$.

We computed another $\chi^{2}$ statistic using the Minkowski functionals for the dust analysis. In this case

$\chi^{2}\left(\alpha_{\mathrm{d}}\right)=\sum_{i, j}\left(\boldsymbol{v}(i)-\langle\boldsymbol{v}(i)\rangle_{\alpha_{\mathrm{d}}}\right) C_{i j}^{-1}\left(\boldsymbol{v}(j)-\langle\boldsymbol{v}(j)\rangle_{\alpha_{\mathrm{d}}}\right)$

$i$ and $j$ cover 11 thresholds from $-2.5 \sigma$ to $2.5 \sigma$ and the three Minkowski functionals. $\langle\boldsymbol{v}(i)\rangle_{\alpha_{\mathrm{d}}}$ is the mean value of the corresponding functional at the corresponding threshold for Gaussian CMB simulations with noise plus $\alpha_{\mathrm{d}}$ times the dust template. $C$ is the covariance matrix for Gaussian CMB simulations with noise. The value of $\alpha_{\mathrm{d}}$ that best fits Archeops data is $\alpha_{\mathrm{d}}=0.0$. Analysing Gaussian simulations without dust we find that $\alpha_{\mathrm{d}} \leq$ 0.28 for $90 \% \mathrm{CL}$, and $\alpha_{\mathrm{d}} \leq 0.35$ for $95 \% \mathrm{CL}$.

\subsection{Primordial non-Gaussianity}

There are several possible inflationary scenarios in which the primordial fluctuations are not Gaussian distributed. The idea is to work with a simple non-Gaussianity model and to impose some constraints on it. In particular, we consider the "weak nonlinear coupling case" (Komatsu \& Spergel 2001; Liguori et al. 2003; Bartolo et al. 2004)

$\Phi(\boldsymbol{x})=\Phi_{L}(\boldsymbol{x})+f_{\mathrm{nl}}\left\{\Phi^{2}(\boldsymbol{x})-\left\langle\Phi^{2}(\boldsymbol{x})\right\rangle\right\}$

where $\Phi(\boldsymbol{x})$ is the primordial gravitational potential, (which satisfies $\langle\Phi(\boldsymbol{x})\rangle=0), \Phi_{L}(\boldsymbol{x})$ is the linear random component (Gaussian distributed), and $f_{\mathrm{nl}}$ is the non-linear dimensionless ${ }^{4}$ coupling parameter.

Scales larger than 1 degree are larger than the horizon scale at the recombination time, when CMB was formed (Liddle \& Lyth 2000). In this regime it is possible to make a good approximation linking CMB fluctuations and gravitational fluctuations through the Sachs-Wolfe effect (Sachs \& Wolfe 1967) $\Delta T(\boldsymbol{n}) / T=\Phi(\boldsymbol{n}) / 3$ (notice however that a better approximation should include the integrated Sachs-Wolfe effect).

\footnotetext{
${ }^{4}$ We use the units system with $c=1$.
}

We analysed signal plus noise simulations with a $f_{\mathrm{nl}}$ term in this way,

$$
\begin{aligned}
\Delta T_{\mathrm{s}}^{\prime}(\boldsymbol{n}) & =\Delta T_{\mathrm{s}}(\boldsymbol{n})+\frac{3 f_{\mathrm{nl}}}{T}\left\{\Delta T_{\mathrm{s}}(\boldsymbol{n})^{2}-\left\langle\Delta T_{\mathrm{s}}(\boldsymbol{n})^{2}\right\rangle\right\} \\
\Delta T(\boldsymbol{n}) & =\Delta T_{\mathrm{s}}^{\prime}(\boldsymbol{n})+\Delta T_{\mathrm{n}}(\boldsymbol{n})
\end{aligned}
$$

where $\Delta T_{\mathrm{s}}$ is a Gaussian signal simulation, $\Delta T_{\mathrm{n}}$ is a Gaussian noise simulation, $T=2.725 \mathrm{~K}$ and $\Delta T$ is the analysed simulation.

We performed a $\chi^{2}$ analysis for the primordial nonGaussianity similar to the dust case for both $U_{2}$ and the Minkowski functionals. The signal-to-noise eigenmodes $y_{i}$ are weakly dependent on $f_{\mathrm{nl}}$. It can be seen that the mean value of $y_{i}^{2}$ for simulations with $f_{\mathrm{nl}}$ is

$$
\begin{aligned}
\left\langle y_{i}^{2}\right\rangle_{f_{\mathrm{nl}}} & =1+\frac{a_{i}}{1+\left(D_{A}\right)_{i}} * f_{\mathrm{nl}}+\frac{b_{i}}{1+\left(D_{A}\right)_{i}} * f_{\mathrm{nl}}^{2} \\
a_{i} & =\frac{1}{T} \sum_{j, k}\left(R_{A}^{t} L_{N}^{-1}\right)_{i j}\left(\left\langle s_{j} s_{k}^{2}\right\rangle+\left\langle s_{k} s_{j}^{2}\right\rangle\right)\left(L_{N}^{-t} R_{A}\right)_{k i} \\
b_{i} & =\frac{1}{T^{2}} \sum_{j, k}\left(R_{A}^{t} L_{N}^{-1}\right)_{i j}\left(\left\langle s_{j}^{2} s_{k}^{2}\right\rangle+\left\langle s^{2}\right\rangle^{2}\right)\left(L_{N}^{-t} R_{A}\right)_{k i}
\end{aligned}
$$

where $b_{i}$ is about an order of magnitude larger than $a_{i}$ for most of the $s / n$ eigenmodes. This implies that $\left\langle y_{i}^{2}\right\rangle_{f_{\mathrm{nl}}}-1 \sim O\left(f_{\mathrm{nl}}^{2}\right)$ which explains the low sensitivity of $U_{2}$ to $f_{\mathrm{nl}}$ variations. In particular, we found that it is much less sensitive than the Minkowski functionals. If we consider for example, a value of $f_{\mathrm{nl}}=2300$, we find a relative variation $\left(\left\langle y_{i}^{2}\right\rangle_{f_{\mathrm{nl}}}-\left\langle y_{i}^{2}\right\rangle_{0}\right) /\left\langle y_{i}^{2}\right\rangle_{0} \simeq 0.05$ (and therefore a similar ratio for $U_{2}$ and $\left.U_{2}^{2}\right)$ for the former and $\left(\left\langle F^{2}\right\rangle_{f_{\mathrm{nl}}}-\left\langle F^{2}\right\rangle_{0}\right) /\left\langle F^{2}\right\rangle_{0} \simeq 0.50$ for the latter.

Therefore we performed a $\chi^{2}$ test with the three Minkowski functionals using different thresholds between $-2.5 \sigma$ and $2.5 \sigma$. In the left panel of Fig. 14 we present the $\chi^{2}$ value of the data for different $f_{\mathrm{nl}}$ cases. We can see that the minimum $\chi^{2}$ value is reached for $f_{\mathrm{nl}}=200$. Taking into account also the results obtained when analysing Gaussian simulations (right panel; Fig. 14) we can put the following constraints on $f_{\mathrm{nl}}$ from the Archeops data: $f_{\mathrm{nl}}=200_{-300}^{+600}$ at $68 \% \mathrm{CL}, f_{\mathrm{nl}}=200_{-600}^{+900}$ at $90 \% \mathrm{CL}$, and $f_{\mathrm{nl}}=200_{-800}^{+1100}$ at $95 \% \mathrm{CL}$. 


\section{Complementary analysis: WMAP in the same region}

WMAP is a NASA satellite dedicated to observe the anisotropies of the CMB with high accuracy at five different frequencies between 23 and $94 \mathrm{GHz}$. Scientific results of this mission have provided us a clearer image of the early universe, and reduced the uncertainties in several cosmological parameters. Data products of this mission can be found on the web $^{5}$.

\subsection{The WMAP data}

We have analysed WMAP data with the same goodness-of-fit and the Minkowski functionals tests already used on Archeops data. The main purpose of this analysis is to compare Archeops results with a different experiment to discriminate among systematics, foreground emissions and intrinsic CMB non-Gaussian features. It is clear that the WMAP frequencies complement very well those of Archeops. A detailed analysis of the possible WMAP non-Gaussianities with this goodness-of-fit method deserves further study.

The maps we analysed were produced from the 1-year and 3-year WMAP foreground cleaned maps for the differencing assemblies corresponding to the cosmological frequencies 40,60 and $90 \mathrm{GHz}$. The main properties of these maps are described in detail in Bennett et al. (2003a) and Hinshaw et al. (2007) respectively.

Specifically we have used the "combined map" as described in Bennett et al. (2003a), (see also Vielva et al. 2004). The WMAP CMB simulations which are used in the analysis are also combined simulations, that is, CMB signal simulations were produced for each channel and then combined in the same manner as for the data.

According to Bennett et al. (2003a), WMAP noise is highly uncorrelated, that is, the noise from a given pixel $i$ is independent of the noise from another pixel $j$. The noise combined simulations are produced from the "combined variance map" as shown for example in Vielva et al. (2004).

We have analysed both combined maps, 1-year and 3-year (hereafter WCM1 and WCM3). The WMAP mask considered for both analyses was the 3-year Kp0 one because it is the most conservative for WCM3 and also contains the 1-year Kp0 mask. See Hinshaw et al. (2007) for details about new masks and Bennett et al. (2003b) for original masks. The actual mask we used is the 3-year WMAP Kp0 degraded to our resolution times the Archeops mask ${ }^{6}$. Its number of pixels is 1648 . In Fig. 15 WCM3 data is plotted using this mask.

\subsection{Gaussianity test on WMAP data}

In order to perform the R\&BT test on WCM1 and WCM3 maps we followed the same steps as for the Archeops analysis. We calculated their corresponding $S$ and $N$ matrices for the 1648 pixels available after applying the combined Archeops-WMAP mask.

We assume the best fit model of the 3-year WMAP data for both analysis, WCM1 and WCM3. At the resolution with which we are dealing, 1.8 degrees, the power spectra of the 1-year and 3 -year data are very approximately the same. This assumption implies that the $S$ matrix is the same for both releases. The $S$

\footnotetext{
5 http://lambda.gsfc.nasa.gov/

6 For comparison, we also repeated the goodness-of-fit analysis on Archeops data using this combined mask, finding similar results to those obtained in Sect. 5 using the Archeops mask.
}

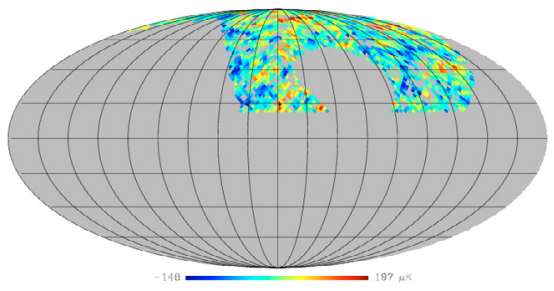

Fig. 15. WCM3 Data at HEALPix resolution $N_{\text {side }}=32$ (it corresponds to a pixel size of $\approx 1.8$ degrees). This map is centred on Galactic longitude $l=180$ degrees. The pixels contaminated by Galactic and extragalactic emission are covered with the mask described in the text. Grid lines are spaced by 20 degrees.

Table 4. Mean and dispersion of $U_{i}^{2}$ statistics from $10^{3}$ WCM1 simulations for $(s / n)_{\mathrm{c}}=3.64$.

\begin{tabular}{c|ccccc|c}
\hline \hline$\ldots$ & $U_{1}^{2}$ & $U_{2}^{2}$ & $U_{3}^{2}$ & $U_{4}^{2}$ & $U_{5}^{2}$ & $\chi_{1}^{2}$ \\
\hline$\mu$ & 1.09 & 1.15 & 1.02 & 1.09 & 1.02 & 1.00 \\
$\sigma$ & 1.56 & 1.50 & 1.47 & 1.71 & 2.02 & 1.41 \\
\hline
\end{tabular}

Table 5. Mean and dispersion of $U_{i}^{2}$ statistics from $10^{3}$ WCM3 simulations for $(s / n)_{\mathrm{c}}=6.33$.

\begin{tabular}{c|ccccc|c}
\hline \hline$\ldots$ & $U_{1}^{2}$ & $U_{2}^{2}$ & $U_{3}^{2}$ & $U_{4}^{2}$ & $U_{5}^{2}$ & $\chi_{1}^{2}$ \\
\hline$\mu$ & 1.00 & 1.18 & 1.04 & 1.10 & 1.22 & 1.00 \\
$\sigma$ & 1.42 & 1.56 & 1.51 & 1.56 & 2.81 & 1.41 \\
\hline
\end{tabular}

matrix is computed from $1.2 \times 10^{5}$ Gaussian simulations according to Eq. (6). Each simulation was produced in the same 90 dual core processors mentioned previously, and took an average CPU time of $360 \mathrm{~s}$ and an average RAM memory of $0.4 \mathrm{~GB}$.

As commented above, WMAP noise is highly uncorrelated and therefore we can assume that the noise matrices are diagonal. This means that the correlation element corresponding to pixels $i$ and $j$ is $N_{i j}=\sigma_{i}^{2} * \delta_{i j}$, where $\sigma_{i}^{2}$ is the combined noise of pixel $i$. Noise matrices for WCM1 and WCM3 must be constructed with their corresponding noise variances which differ by an approximate factor of 3 .

Two additional sets of $10^{3}$ Gaussian signal plus noise simulations (corresponding to WCM1 and WCM3 maps) were performed for the calibration of the matrices. In Fig. 16, we present the mean and the dispersion of the $U_{i}^{2}$ statistics at different signal-to-noise cuts for the WCM3 case. Note that the numerical range for the possible signal-to-noise cuts $(s / n)_{\mathrm{c}}$ is wider than for the Archeops case, because WCM3 noise is smaller than that of Archeops at this resolution. The $(s / n)_{\mathrm{c}}$ range for WCM1 is approximately the same as that of WCM3 reduced by a factor $\sqrt{3}$. The mean and the dispersion for WCM1 simulations are similar to those obtained for WCM3. It can be seen that mean values of $U_{i}^{2}$ statistics are close to 1 for almost all signal-to-noise cuts and all the computed statistics, but the dispersion becomes higher than square root of two for high signal-to-noise cuts and for statistics with high order moments, such as $U_{5}^{2}$ and higher order statistics.

As for the Archeops case, these high values are explained by the small errors present in the computed correlation matrices plus small numerical errors in the diagonalisation of these matrices, which are amplified through the high order moments. In Table 4 we present the mean and the dispersion of $U_{i}^{2}$ statistics for $10^{3}$ WCM1 simulations with noise for all the eigenmodes $(s / n \geq 3.64)$. Note how the dispersion increases with the order of the statistics. In Table 5 the same quantities are presented 

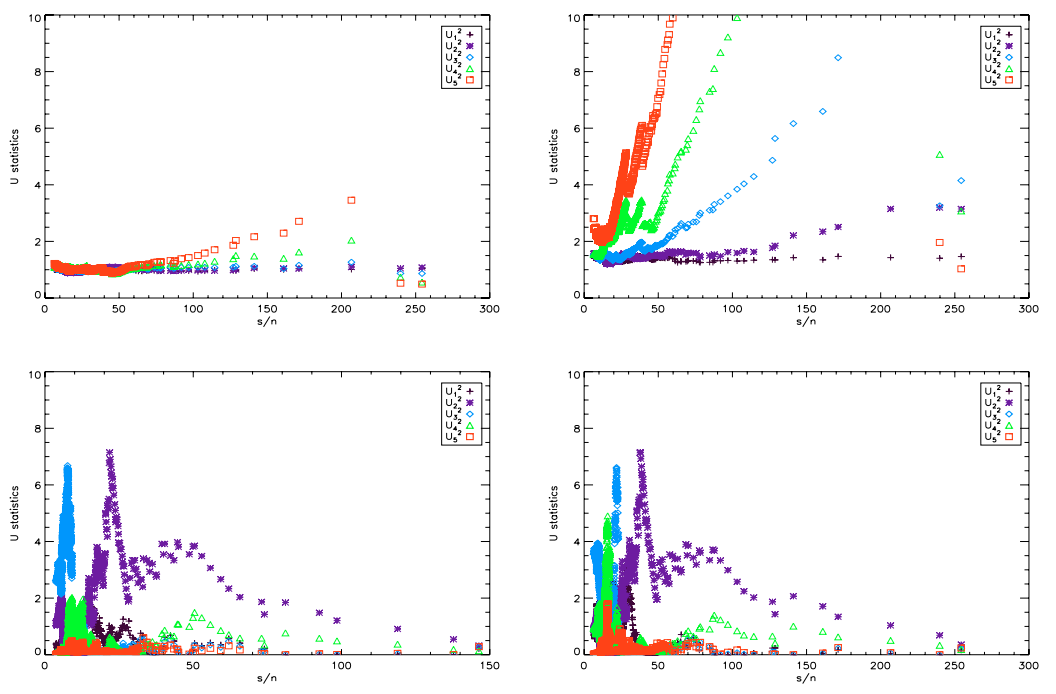

Fig. 16. From left to right, mean and dispersion of $U_{i}^{2}$ statistics (where $i$ goes from 1 to 5) for different signal-to-noise cuts, corresponding to $10^{3}$ signal plus noise WCM3 simulations.

Fig. 17. From left to right, $U_{i}^{2}$ statistics for WCM1 and WCM3 presented for different signal-to-noise cuts.
Table 6. WCM1 $U_{i}^{2}$ statistics for $(s / n)_{\mathrm{c}}=21.81$, and their corresponding upper tail probabilities.

\begin{tabular}{c|ccccc}
\hline \hline$\ldots$ & $U_{1}^{2}$ & $U_{2}^{2}$ & $U_{3}^{2}$ & $U_{4}^{2}$ & $U_{5}^{2}$ \\
\hline WCM1 & 0.90 & 7.15 & 0.32 & 0.63 & 0.09 \\
Prob. & 0.37 & 0.01 & 0.52 & 0.35 & 0.67 \\
\hline
\end{tabular}

Table 7. WCM $3 U_{i}^{2}$ statistics for $(s / n)_{\mathrm{c}}=37.92$, and their corresponding upper tail probabilities.

\begin{tabular}{c|ccccc}
\hline \hline$\ldots$ & $U_{1}^{2}$ & $U_{2}^{2}$ & $U_{3}^{2}$ & $U_{4}^{2}$ & $U_{5}^{2}$ \\
\hline WCM3 & 0.13 & 7.15 & 0.00 & 0.61 & 0.01 \\
Prob. & 0.73 & 0.01 & 0.95 & 0.36 & 0.88 \\
\hline
\end{tabular}

for $10^{3}$ WCM3, obtained also from all the eigenmodes $(s / n \geq$ $6.33)$. The effect is the same for the high order moment statistics. The results for the $U_{i}^{2}$ statistics for WCM1 and WCM3 data maps are presented in Fig. 17. As can be seen, all $U_{i}^{2}$ values satisfy $U_{i}^{2} \leq 7.15$. The upper limit 7.15 corresponds to a upper tail probability of $0.7 \%$ for the theoretical distribution. To confirm or rule out a possible non-Gaussian detection, this result should be studied more carefully. Firstly, we have that for both WCM1 and WCM3 $U_{2}^{2}$ is the only statistic which reaches some sharp peaks above 6.6 (which corresponds to a upper tail probability for the theoretical distribution of $1.0 \%$ ). From the plots in Fig. 17, $U_{2}^{2}$ reaches this peak at $(s / n)_{\mathrm{c}}=21.81$ for WCM1 and $(s / n)_{\mathrm{c}}=37.92$ for WCM3. We estimated the upper tail probability for the $U_{i}^{2}$ statistics of the data at the mentioned signal-tonoise cut by performing $10^{3}$ Gaussian simulations. These results are presented in Tables 6 and 7. As we can see for the $U_{2}^{2}$ statistic, we have this probability as $1.0 \%$ and $0.7 \%$ for WCM1 and WCM3 respectively, very similar to the theoretical value.

This probability is obtained for the precise signal-to-noise cut where $U_{2}^{2}$ reaches its maximum. Since the width of the maxima is much smaller than the range of variation of the signal-to-noise eigenvalues, it makes sense to ask for the significance of the detection. Thus, from the simulations we computed the " $p$-value", i.e. the probability of finding a value of $U_{2}^{2}$ larger than 7.15 at any signal-to-noise cut, the maximum value reached by the data. This probability is $18 \%$ for WCM1 and $17 \%$ for WCM3.

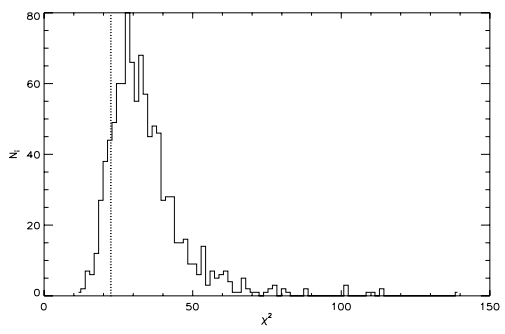

Fig. 18. Distribution of the $\chi^{2}$ values from the Minkonwski Gaussian test for WCM3 data. Vertical line shows the data results. Their cumulative probability is $12.0 \%$.

From the previous discussion, we conclude that the sharp peaks found in the data are not significant. Also, well studied cases of artificial CMB non-Gaussianities, such as skewness or kurtosis produced using the Edgeworth expansion (Martínez-González et al. 2002, for applications of this expansion to the CMB non-Gaussianity analyses), usually show deviations of the $U_{i}^{2}$ statistics in the form of a large plateau. Besides, we note that at the signal-to-noise cuts where the maxima are found, there are fewer than $100\left\{y_{i}\right\}$ numbers to compute the $U_{i}^{2}$ statistics (around 70), and the test only works correctly asymptotically $(n \gg 1)$.

WCM3 data were also analysed with the Minkowski functionals as in the Archeops case (that is, using 11 thresholds between $-2.5 \sigma$ and $2.5 \sigma$ and the three functionals). The histogram corresponding to the $\chi^{2}$ values for 1000 Gaussian simulations and the value for WCM3 data are presented in Fig. 18. As we can see, the WCM3 data are again compatible with Gaussianity.

Finally, we performed an analysis on simulations with the $f_{\mathrm{nl}}$ parameter as defined in Eq. (15). The procedure was the same as that performed for Archeops case. As discussed in Sect. 5.2, we only use the Minkowski functionals for the $f_{\mathrm{nl}}$ case. The $\chi^{2}$ value for WCM3 data is minimum for $f_{\mathrm{nl}}=100$. Analysing Gaussian simulations, the constraints found for $f_{\mathrm{nl}}$ are: $f_{\mathrm{nl}}=100_{-200}^{+200}$ at $68 \% \mathrm{CL}, f_{\mathrm{nl}}=100_{-300}^{+400}$ at $90 \% \mathrm{CL}, f_{\mathrm{nl}}=100_{-400}^{+500}$ at $95 \% \mathrm{CL}$. These limits are compatible with those obtained from Archeops since the tighter constraints found for WCM3 can be explained by the significantly smaller noise in that experiment. In particular, if we analyse simulated Archeops data with noise normalised to the same amplitude as that of WCM3 we find similar limits for $f_{\mathrm{nl}}$. 


\section{Conclusions}

The expected behaviour of the $U_{i}^{2}$ statistics as a $\chi_{1}^{2}$ distribution has been confirmed for the order index interval $1 \leq i \leq 4$ with "realistic" simulations assuming Gaussian CMB anisotropies. For higher moments, $i>4$, the mean of the distribution is $\mu \simeq 1$ but the variance is $\sigma \gtrsim 2$. This is because of the propagation of errors through higher order moments which in practice complicates the use of high order $U_{i}^{2}$ in our analysis.

From the analysis of both kinds of Archeops maps, coaddition and Mirage, we have found that both are compatible with Gaussianity. Only the $U_{2}^{2}$ statistic for coaddition map is close to 8.0 for low $(s / n)_{\mathrm{c}}$. Although in principle the probability that $U_{2}^{2}$ takes values greater than 8.0 for a given signal-to-noise cut in the Gaussian hypothesis is very low (see Table 3), the corresponding " $p$-value" for having $U_{2}^{2}$ larger than 8.0 at any signalto-noise cut is 0.1482 . This is not negligible and thus this detection is not significant. Moreover this effect does not appear in the Mirage map, and therefore should be assigned to issues related to the map-making process.

The analysis with the Minkowski functionals on the Mirage map also returns compatibility with Gaussianity.

Our analysis also implies constraints on the amount of contamination that can be present at $143 \mathrm{GHz}$. Using as template for dust and atmosphere the Archeops map at $353 \mathrm{GHz}$, we limit the possible contamination to be lower than $7.8 \%$ at $90 \%$ CL using $U_{2}$ statistic. A similar limit is obtained with the Minkowski functionals.

We also compared the Archeops results with the WMAP 1 and 3-year data in the same region of the sky. For both sets of data, a sharp peak in $U_{2}^{2}$ was found at specific signal-tonoise cuts. Although the probability of finding such a peak at a given signal-to-noise cut is very small, the " $p$-value" obtained when different cuts are allowed is appreciable. Therefore we can conclude that the WMAP data, when the same region as Archeops is considered, are also consistent with Gaussianity. The same conclusion is reached when the data are analysed with the Minkowski functionals.

Finally, we established a constraint in the value of the nonlinear coupling parameter $f_{\mathrm{nl}}$. Analysing Archeops data, we found that $f_{\mathrm{nl}}=200_{-600}^{+900}$ at $90 \% \mathrm{CL}$, and $f_{\mathrm{nl}}=200_{-800}^{+1100}$ at $95 \%$ CL. When the same analysis was done with WMC3 data using Archeops-WMAP combined mask, we found $f_{\mathrm{nl}}=100_{-300}^{+400}$ at $90 \% \mathrm{CL}, f_{\mathrm{nl}}=100_{-400}^{+500}$ at $95 \% \mathrm{CL}$. These limits are similar to those expected for an Archeops-like experiment with a noise amplitude similar to that of WCM3.

Acknowledgements. The authors kindly thank the Archeops Collaboration for the possibility of using Archeops data. We are especially grateful for the access to the computational resources of the IFCA Computing Group. A.C. thanks the Spanish Ministerio de Educación y Ciencia (MEC) for a pre-doctoral FPI fellowship, P. Vielva for his help and comments on the simulation of WMAP, and A. M. Aliaga for useful discussions on the Smooth tests of goodness-of-fit.

We acknowledge partial financial support from the Spanish MEC project ESP2004-07067-C03-01 and the joint project CSIC-CNRS, with reference 2004FR009. We acknowledge the use of LAMBDA. Support for it is provided by the NASA Office of Space Science. We used the CAMB code (Lewis et al. 2000 ) for our analysis. The CAMB code is derived from CMBFAST (Zaldarriaga \& Seljak 2000). The HEALPix package was used throughout the data analysis (Górski et al. 2005).

\section{References}

Aliaga, A. M., Martínez-González, E., Cayón, L., et al. 2003, New Astron. Rev., 47, 907

Aliaga, A. M., Rubiño-Martín, J. A., Martínez-González, E., Barreiro, R. B., \& Sanz, J. L. 2005, MNRAS, 356, 1559
Barreiro, R. B., Hobson, M. P., Lasenby, A. N., et al. 2000, MNRAS, 318, 475 Barreiro, R. B., Martínez-González, E., \& Sanz, J. L. 2001, MNRAS, 322, 411 Barreiro, R. B., Rubiño-Martín, J. A., \& Martínez-González, E. 2007, Highlights of Spanish Astrophysics IV, ed. F. Figueras, J. M. Girart, M. Hernanz, \& C. Jordi (Springer), Proceedings of the VII Scientific Meeting of the Spanish Astronomical Society (SEA), held in Barcelona, September 12-15, 2006 Bartolo, N., Komatsu, E., Matarrese, S., \& Riotto, A. 2004, Phys. Rep., 402, 103 Bennett, C. L., Halpern, M., Hinshaw, G., et al. 2003a, ApJ, 148, 1 Bennett, C. L., Hill, R. S., Hinshaw, G., et al. 2003b, ApJ, 148, 97 Benoît, A., Ade, P., Amblard, A., et al. 2002, Astropart. Phys., 17, 101 Benoît, A., Ade, P., Amblard, A., et al. 2003a, A\&A, 399, L19

Benoît, A., Ade, P., Amblard, A., et al. 2003b, A\&A, 399, L25

Benoît, A., Ade, P., Amblard, A., et al. 2004, A\&A, 424, 571

de Bernardis, P., Ade, P., Bock, J. J., et al. 2000, Nature, 404, 955

Bond, J. R. 1995, Phys. Rev. Lett., 74, 4369

Cayón, L., Martínez-González, E., Argüeso, F., Banday, A. J., \& Górski, K. M. 2003a, MNRAS, 339, 1189

Cayón, L., Argüeso, F., Martínez-González, E., \& Sanz, J. L. 2003b, MNRAS, 344,917

Copi, C. J., Huterer, D., \& Starkman, G. D. 2004, Phys. Rev. D, 70, 043515

Copi, C. J., Huterer, D., Schwarz, D. J., \& Starkman, G. D. 2006, MNRAS, 367, 79

Cruz, M., Martínez-González, E., Vielva, P., \& Cayón, L. 2005, MNRAS, 356, 29

Cruz, M., Tucci, M., Martínez-González, E., \& Vielva, P. 2006, MNRAS, 369, 57

Cruz, M., Cayón, L., Martínez-González, E., Vielva, P., \& Jin, J. 2007, ApJ, 655, 11

Eriksen, H. K., Hansen, F. K., Banday, A. J., Górski, K. M., \& Lilje, P. B. 2004, ApJ, 605, 14

Eriksen, H. K., Banday, A. J., Górski, K. M., \& Lilje, P. B. 2005, ApJ, 622, 58 Ferreira, P. G., Magueijo, J., \& Silk, J. 1997, Phys. Rev. D, 56, 4592 Ferreira, P. G., Magueijo, J., Górski, K. M., \& Krzysztof, M. 1998, ApJ, 503, L1 Górski, K. M., Hivon, E., Banday, A. J., et al. 2005, ApJ, 622, 759 Gott III, J. R., Park, C., Juszkiewicz, R., et al. 1990, ApJ, 352, 1 Guth, A. H. 1981, Phys. Rev. D, 23, 347

Halverson, N. W., Leitch, E. M., Pryke, C., et al. 2002, ApJ, 568, 38

Hanany, S., Ade, P., Balbi, A., et al. 2000, ApJ, 545, 5

Hinshaw, G., Nolta, M. R., Bennet, C. L., et al. 2007, ApJ, 170, 288

Hobson, M. P., Jones, A. W., \& Lasenby, A. N. 1999, MNRAS, 309, 125

Komatsu, E., \& Spergel, D. N. 2001, Phys. Rev. D, 63, 063002

Komatsu, E., Kogut, A., Nolta, M. R., et al. 2003, ApJS, 148, 119

Lamarre, J. M., Puget, J. L., Bouchet, F., et al. 2003, New Astron. Rev., 47, 1017 Larson, D. L., \& Wandelt, B. D. 2004, ApJ, 613, 85

Lewis, A., Challinor, A., \& Lasenby, A. 2000, ApJ, 538, 473

Liddle, A. R., \& Lyth, D. H. 2000, Cosmological Inflation and Large-Scale Structure (Cambridge: Cambridge University Press)

Liguori, M., Matarrese, S., \& Moscardini, L. 2003, ApJ, 597, 57

Linde, A. D. 1990, Particle Physics and Inflationary Cosmology (Hardwood, Chur)

Lyth, D. H., \& Riotto, A. 1998, Phys. Rept., 314, 1

Macías-Pérez, J. F., Lagache, G., Maffei, B., et al. 2007, A\&A, 467, 1313

Magueijo, J. 2000, ApJ, 528, L57

Martínez-González, E., Gallegos, J. E., Argüeso, F., \& Sanz, J. L. 2002, MNRAS, 336, 22

Monteserín, C., Barreiro, R. B., Sanz, J. L., \& Martínez-González, E. 2005, MNRAS, 360, 9

Monteserín, C., Barreiro, R. B., Martínez-González, E., \& Sanz, J. L. 2006, MNRAS, 371, 312

Ponthieu, N., Macías-Pérez, J. M., Tristram, M., et al. 2005, A\&A, 444, 327

Rayner, J. C. W., \& Best, D. J. 1989, Smooth Tests of Goodness of Fit (New York: Oxford University Press)

Rayner, J. C. W., \& Best, D. J. 1990, Int. Stat. Rev., 58, 9

Rubiño-Martín, J. A., Aliaga, A. M., Barreiro, R. B., et al. 2006, MNRAS, 369, 909

Sachs, R. K., \& Wolfe, A. M. 1967, ApJ, 147, 73

Schmalzing, J., \& Górski, K. M. 1998, MNRAS, 297, 355

Spergel, D. N., Bean, R., Doré, O., et al. 2007, ApJ, 170, 377

Smoot, G. F, Bennett, C. L., Kogut, A., et al. 1992, ApJ, 396, 1

Tristram, M., Patanchon, G., Macías-Pérez, J. F., et al. 2005, A\&A, 436, 785

de Troia, G., Ade, P., Bock, J. J., et al. 2007 [arXiv : 0705. 1615v1]

Vielva, P., Martínez-González, E., Barreiro, R. B., Sanz, J. L., \& Cayón, L. 2004, ApJ, 609, 22

Yvon, D., \& Mayet, F. 2005, A\&A, 436, 729

Wiaux, Y., Vielva, P., Martínez-González, E., \& Vandergheynst, P. 2006, Phys. Rev. Lett., 96, 151303

Zaldarriaga, M., \& Seljak, U. 2000, ApJS, 129, 431 